\title{
AN INFORMAL ARITHMETICAL APPROACH TO COMPUTABILITY AND COMPUTATION, II
}

\author{
Z.A. Melzak \\ (received July 11, 1963)
}

11. In the first part of this paper [1] there was introduced a hypothetical computing device, the Q-machine. It was derived by abstracting from the process of calculating carried out by a man on his fingers, assuming an adequate supply of hands and the ability to grow fingers at will. The Q-machine was shown to be equal in computing power to a universal Turing machine. That is, the $Q$-machine could compute any number regarded as computable by any theory of computability developed so far. It may be recalled here that Turing machines were obtained by Turing [2] by abstracting from the process of calculating carried out by a man on some concrete 'symbol space' (tape, piece of paper, blackboard) by means of fixed but arbitrary symbols. Hence the contrast between the $Q$-machine and the Turing machines is that between arithmetical manipulation of counters and logical manipulation of symbols. In particular, one might say, loosely, that in a Turing machine, as in arithmetic, numbers are represented by signs whereas in the $Q$-machine, as on a counting frame, numbers represent themselves.

The programs of the $\mathrm{Q}$-machine were written in terms of a ternary command scheme of the type

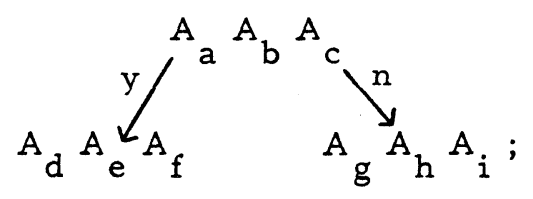

when that is executed, the contents $p, q, r$ of the locations $A_{a}, A_{b}, A_{c}$ become respectively $p-q \operatorname{sgn}(p-q), q, r+q \operatorname{sgn}(p-q)$.

Canad. Math. Bull. vol. 7, no. 2, April 1964 
Here $\operatorname{sgn} x$ is defined to be 1 if $x \geq 0$, and to be 0 otherwise.

The following generalization suggests itself: let $n$ be a positive integer, $n \geq 2$, and let $f_{i}\left(x_{1}, \ldots, x_{n}\right), i=1, \ldots, n$, be $\mathrm{n}$ integer-valued functions, each one defined for all nonnegative values of its integer arguments; now replace the scheme (1) by

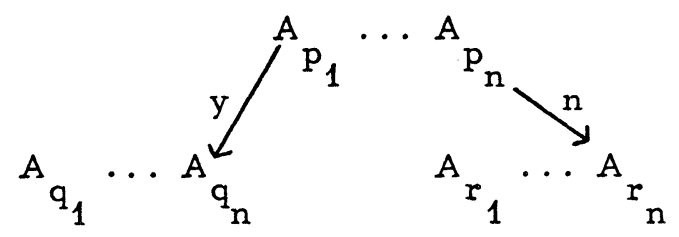

which is to be executed as follows: Let $c_{1}, \ldots, c_{n}$ be the contents of the locations $\mathrm{A}_{\mathrm{p}_{1}}, \ldots, \mathrm{A}_{\mathrm{p}_{\mathrm{n}}} ;$ if

$$
c_{i}+f_{i}\left(c_{1}, \ldots, c_{n}\right) \geq 0, \quad i=1, \ldots, n
$$

then the contents $c_{1}, \ldots, c_{n}$ are changed to $c_{1}+f_{1}\left(c_{1}, \ldots, c_{n}\right)$, $\ldots, c_{n}+f_{n}\left(c_{1}, \ldots, c_{n}\right)$ respectively, and the next command to be carried out is $A_{q_{1}} \ldots A_{q_{n}}$. If for some $i, c_{i}+f_{i}\left(c_{1}, \ldots, c_{n}\right)<0$ then the contents $c_{1}, \ldots, c_{n}$ are left unchanged and the next command to be carried out is $A_{r_{1}} \ldots A_{r_{n}}$. All previous conventions regarding subscripted locations ${ }_{\mathrm{A}}^{\mathrm{A}_{\mathrm{m}}}$, program loops, sub-programs, stops etc. remain in force. Any such machine will be called a $Q$-machine (or a $Q_{n}$-machine, or the machine $\left.Q_{n}\left(f_{1}, \ldots, f_{n}\right)\right), n$ will be called its degree, and the functions $\mathrm{f}_{1}, \ldots, \mathrm{f}_{\mathrm{n}}$ its transfer functions. Individual $\mathrm{Q}$-machines will be distinguished by superscripts; thus $Q_{3}^{0}$ or $Q_{3}^{0}\left(f_{1}, f_{2}, f_{3}\right)$, with $f_{1}\left(c_{1}, c_{2}, c_{3}\right)=-f_{3}\left(c_{1}, c_{2}, c_{3}\right)=-c_{2}, f_{2}=0$, will be the old Q-machine of the first part of this paper. We make one final proviso: in (2) the commands may contain the store symbol $S$ 
once or more times, but not so as to involve the transfer of infinitely many counters; also, no command should be such that for any contents of the locations involved no counters get transferred. Thus the $Q_{3}^{0}$ commands $S A_{i}$ or $A_{i} S A_{j}$ are excluded. When the first command in (2) is carried out, the original contents $c_{1}, \ldots, c_{n}$ are changed to

$$
c_{i}+f_{i}\left(c_{1}, \ldots, c_{n}\right) \prod_{j=1}^{n} \operatorname{sgn}\left[c_{j}+f_{j}\left(c_{1}, \ldots, c_{n}\right)\right], \quad i=1, \ldots, n \text {. }
$$

When $n_{1}=n_{2}$ and $f_{i}=g_{i}, i=1, \ldots, n_{1}$, then the Q-machines $Q_{n_{1}}\left(f_{1}, \ldots, f_{n}\right)$ and $Q_{n_{2}}\left(g_{1}, \ldots, g_{n}\right)$ are identical.

However, it may happen that the transfer functions of two Q-machines of the same degree are not the same and yet their effects with any initial location-contents and for any program are the same; consider, for instance, $Q_{3}^{0}$ and the machine $Q_{3}^{1}\left(f_{1}^{\prime}, f_{2}^{\prime}, f_{3}^{\prime}\right)$ with $f_{1}=f_{1}^{\prime}, f_{3}=f_{3}^{\prime}$ and $f_{2}^{\prime}=\operatorname{sgn}\left(c_{2}-c_{1}-1\right)$. The point is that here $f_{2}^{\prime}$ differs from $f_{2}$ only for $c_{2}>c_{1}$, so that $Q_{3}^{1}$ and $Q_{3}^{0}$ are effectively the same.

Many questions may be raised now about the universality, loop types, structure etc. of the Q-machines and some of them will be considered here. The generalization from the old $Q$-machine to the present $Q$-machines is natural if one wishes to investigate the effect of allowing different basic operations in computing. For instance, $Q_{3}^{0}$ is essentially an adder while the machine $Q_{5}^{0}\left(f_{1}, \ldots, f_{5}\right)$, with $f_{2}=f_{3}=0, f_{1}\left(c_{1}, c_{2}, c_{3}, c_{4}, c_{5}\right)$ $=-c_{2}-c_{3}-c_{2} c_{3}, f_{4}\left(c_{1}, c_{2}, c_{3}, c_{4}, c_{5}\right)=c_{2}+c_{3}$, and $f_{5}\left(c_{1}, c_{2}, c_{3}, c_{4}, c_{5}\right)=c_{2} c_{3}$, is essentially an adder-andmultiplier.

12. A $Q$-machine is universal if it can compute any computable number. In this section we describe the machine $Q_{2}^{0}\left(f_{1}, f_{2}\right)$ which has some claim to being the simplest of all 
universal computing devices; here the transfer functions are $f_{1}\left(c_{1}, c_{2}\right)=-f_{2}\left(c_{1}, c_{2}\right)=-1$. In words, there is a binary command scheme

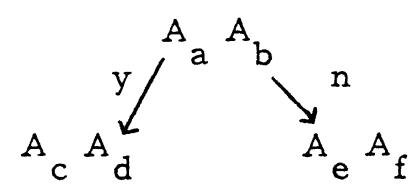

carried out by transferring one counter from the location $\mathrm{A}_{\mathrm{a}}$ to $A_{b}$ if $A_{a}$ is not empty and proceeding to the command $A_{c} A_{d}$, and leaving the contents of $A_{a}$ and $A_{b}$ unchanged and then proceeding to the command $A_{e} A_{f}$ in case $A_{a}$ is empty. It might be assumed that initially all the locations are empty since the $Q_{2}^{0}$ program $\left(S A_{1}\right)^{p}\left(S A_{2}\right)^{q} \ldots\left(S A_{n}\right)^{r}$ transforms the initial contents $0, \ldots, 0, \ldots$ to $p, q, \ldots, r, 0, \ldots$ We note first two simple sub-routines for $Q_{2}^{0}$ :

1) Transfer $T\left(A_{1}, A_{2}\right)$

initial contents: $p, q, 0, \ldots$

program:

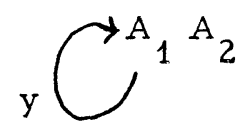

final contents: $0, p+q, 0, \ldots$

This program needs no reserved locations. The special case $T(A, S)$ reads 'empty $A^{\prime}$.

2) Copy $C\left(A_{1}, A_{2}, A_{3}\right)$

initial contents: $p, q, 0,0, \ldots$ 
program:

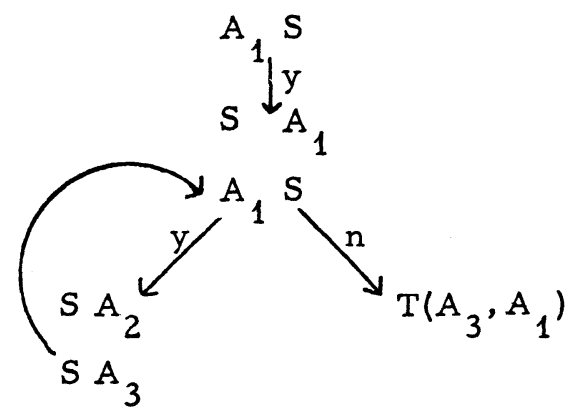

final contents: $p, p+q, 0,0, \ldots$

Now we have the

3) $Q_{3}^{0}-Q_{2}^{0}$ equivalence program

initial contents: $p, q, r, 0,0,0, \ldots$

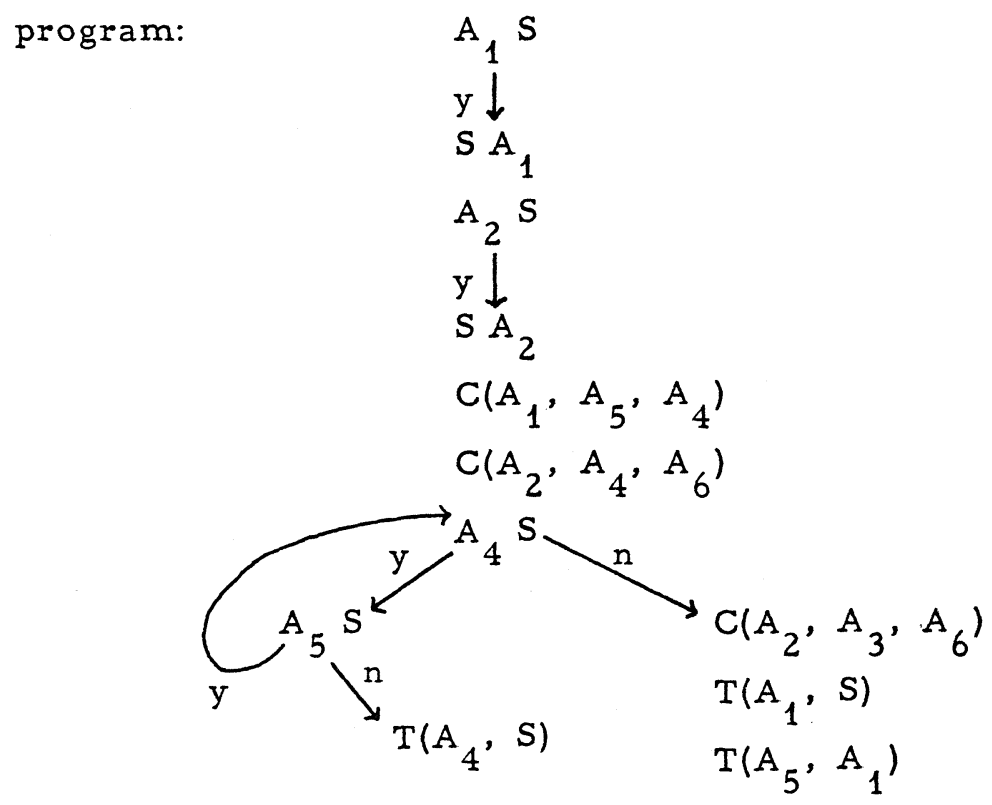

final contents: $p-q \operatorname{sgn}(p-q), q, r+q \operatorname{sgn}(p-q), 0,0,0, \ldots$

This shows that the command $\mathrm{A}_{1} \mathrm{~A}_{2} \mathrm{~A}_{3}$ can be simulated as a program on $Q_{2}^{0}$. Clearly the converse is also true: the command $A_{1} A_{2}$ of $Q_{2}^{0}$ can be simulated as a $Q_{3}^{0}$ program consisting 
of one instruction $A_{1} A_{3} A_{2}$, with the contents of $A_{3}$ equal to 1. Therefore any $Q_{3}^{0}$ program can be translated into its $Q_{2}^{0}$ equivalent and vice versa. Since $Q_{3}^{0}$ has already been shown to be universal, so is $Q_{2}^{0}$.

13. Consider the three already defined $Q$-machines $Q_{2}^{0}, Q_{3}^{0}$ and $Q_{5}^{0}$. They form a hierarchy: $Q_{2}^{0}$ can add 1 , $Q_{3}^{0}$ can add two integers, $Q_{5}^{0}$ can add and multiply two integers. Define $A_{1} A_{2} O_{4} A_{5}$ to be the $Q_{5}^{0}$ command $A_{1} A_{2} A_{3} A_{4} A_{5}$, subject to the condition that the location $A_{3}$ stays empty throughout. The effect of the command $A_{1} A_{2} 0 A_{4} A_{5}$ on the locations $A_{1}, A_{2}$ and $A_{4}$ is the same as that of the $Q_{3}^{0}$ command $A_{1} A_{2} A_{4}$. This leads to a generalization. Let $A_{1} A_{2} \ldots A_{n}$ be the command of a Q-machine and put

$$
P\left(A_{1} A_{2} \ldots A_{n}\right)=A_{1}^{\prime} A_{2}^{\prime} \ldots A_{n}^{\prime},
$$

where each $A_{i}^{\prime}$ is either $A_{i}$ itself or some fixed integer $\mathrm{p}_{i} \geq 0$. In the latter case the location $A_{i}$ retains its contents $p_{i}$ throughout the program. Leaving out all inactive indices (referring to the locations to which fixed contents have been pre-assigned) we have $P\left(A_{1} A_{2} \ldots A_{n}\right)=A_{j_{1}} A_{j_{2}} \ldots A_{j_{N}}, 1 \leq j_{1}<j_{2}<\ldots<j_{N} \leq n$. Now let $\mathrm{m} \leq \mathrm{N}$ and let $\mathrm{i}_{1}, \mathrm{i}_{2}, \ldots, \mathrm{i}_{\mathrm{m}}$ be an increasing subsequence of the sequence $j_{1}, j_{2}, \ldots, j_{N}$. This induces a $Q_{m}$-machine with the command $A_{i_{1}} A_{i_{2}} \ldots A_{i m}$ which we shall call a contraction of the original Q-machine; the latter is called an extension of the former. We note that $Q_{5}^{0}$ is an 
extension of $Q_{3}^{0}$ and of $Q_{2}^{0}$ and $Q_{3}^{0}$ is an extension of $Q_{2}^{0}$, since

$A_{1} A_{2} A_{4}=A_{1} A_{2} 0 A_{4} 0, A_{1} A_{4}=A_{1} 10 A_{4} 0, A_{1} A_{4}=A_{1} 1 A_{4}$.

One Q-machine may be an extension or a contraction of several Q-machines. In fact, it is easy to show that any sequence $\left\{\mathrm{Q}_{\mathrm{n}_{\mathrm{k}}}^{\mathrm{k}}\left(\mathrm{f}_{\mathrm{k} 1}, \ldots, \mathrm{f}_{\mathrm{kn}}\right)\right\}(\mathrm{k}=0,1, \ldots)$ of $\mathrm{Q}$-machines of bounded degrees, $n_{k} \leq N$, possesses a common extension $Q_{N+1}\left(F_{1}, \ldots, F_{N+1}\right)$. For it may be assumed without loss of generality that $\mathrm{n}_{0}=\mathrm{n}_{1}=\ldots=\mathrm{N}$ and then we have only to define $F_{N+1}\left(c_{1}, \ldots, c_{N+1}\right)=0, F_{i}\left(c_{1}, \ldots, c_{N}, k\right)=f_{k i}\left(c_{1}, \ldots, c_{N}\right), i=1, \ldots, N$ to have the desired extension property for $Q_{n_{k}}^{k}: A_{1} A_{2} \ldots A_{N}$ $=\mathrm{A}_{1} \mathrm{~A}_{2} \ldots \mathrm{A}_{\mathrm{N}} \mathrm{k}$.

The following propositions are easily demonstrated: 1) any extension of a universal machine is universal; 2) the set of all Q-machines is uncountable and so is the set of all universal $Q_{n}$-machines for $n \geq 3$; 3) each finite or countably infinite set of $\mathrm{Q}$-machines of degrees $\leq \mathrm{N}$ has uncountably many common extensions which are universal and are of degree $N+1 ; 4)$ the binary relation $Q^{a} \leq Q^{b}\left(Q^{b}\right.$ is an extension of $\left.Q^{a}\right)$ is a partial but not a total order on the set of all $Q$-machines; 5) defining $Q^{a}<Q^{b}$ by $Q^{a} \leq Q^{b}$ and $Q^{b} \ddagger Q^{a}$, we have uncountably many chains $Q^{1}<Q^{2}<\ldots$ starting with any given $Q^{1}$. In each case the uncountable multiplicity follows from the sufficient freedom of choice of values of one or more transfer functions.

14. The set of all $Q$-machines is inconveniently and somewhat unnaturally large, and we should like to limit it somehow. This limitation could be carried out in at least 
two essentially distinct ways. Proceeding 'internally', we may define a number of 'atomic arithmetical operations', such as the decision whether a finite set is empty or not, transferring one member from a non-empty set to another set, merging two finite sets, the decision whether one finite set has more members than another one etc., and then we may consider only those $\mathrm{Q}$-machines whose commands are executable in terms of the se arithmetical operations. This amounts to singling out certain basic functions and allowing only those Q-machines whose transfer functions are (allowable) compositions of the basic ones. The whole procedure may be suitably formalized and one obtains then something similar to the theory of recursive functions.

Proceeding 'externally', we choose a universal machine and we limit ourselves only to those Q-machines whose commands can be synthesized as programs of that universal machine. We shall follow this course and we shall take $Q_{2}^{0}$ as the basic universal machine. That is, in the remainder of this paper, unless the contrary is explicitly stated, we consider only those $Q$-machines with the command $A_{1} A_{2} \ldots A_{n}$, for which a $Q_{2}^{0}$ program $P=P\left(p_{1}, \ldots, P_{M}\right)$ exists, such that the command $A_{1} \ldots A_{n}$ of $Q_{n}$, with the initial contents $p_{1}, \ldots, p_{M}, 0, \ldots$ leads to the same final contents as the $Q_{2}^{0}$ program $P$ (with the initial contents all empty). On the other hand, for ease of description we can use instead of $Q_{2}^{0}$ either $Q_{3}^{0}$ or $Q_{5}^{0}$ or any other Q-machine which has been shown to be equivalent in the above sense to $Q_{2}^{0}$. The equivalence of $Q_{3}^{0}$ and $Q_{2}^{0}$ has been shown, the equivalence of $Q_{5}^{0}$ and $Q_{2}^{0}$ will follow from it and from the $Q_{5}^{0}-Q_{3}^{0}$ equivalence program given below. Since $Q_{5}^{0}$ is an extension of $Q_{3}^{0}$, it suffices to show that the command of $Q_{5}^{0}$ is obtainable as a $Q_{3}^{0}$ program. 
initial contents: $p, q, r, s, t, 1,0,0,0,0, \ldots$

program:

$\mathrm{S}_{1} \mathrm{~A}_{7}$

$\mathrm{S} \mathrm{A}_{2} \mathrm{~A}_{8}$

$\mathrm{S} \mathrm{A}_{3} \mathrm{~A}_{9}$

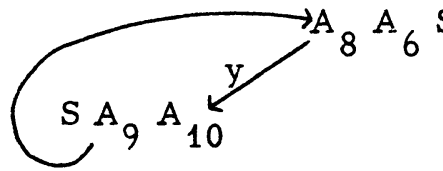

$\mathrm{S}_{\mathrm{S} \mathrm{A}_{2} \mathrm{~A}_{8}}$

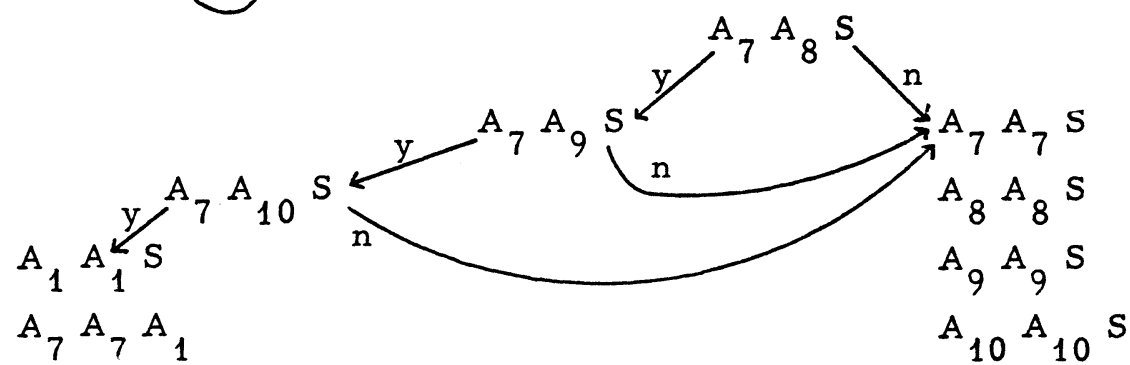

$\mathrm{A}_{8} \mathrm{~A}_{8} \mathrm{~A}_{4}$

$\mathrm{A}_{9} \mathrm{~A}_{9} \mathrm{~A}_{4}$

$\mathrm{A}_{10} \mathrm{~A}_{10} \mathrm{~A}_{5}$

final contents: the same as after the $Q_{5}^{0}$ command

$$
\mathrm{A}_{1} \mathrm{~A}_{2} \mathrm{~A}_{3} \mathrm{~A}_{4} \mathrm{~A}_{5} \text {. }
$$

The set of all admissible Q-machines is now isomorphic to a subset $\jmath_{2}^{0}$ of the set of all $Q_{2}^{0}$ programs. $\zeta_{2}^{0}$ is countable and an explicit enumeration can be given. Recalling the conventions about the arrows, we can put every program $P \in \mathcal{T}_{2}^{0}$ in the form of a string $\mathrm{A}_{\mathrm{a}_{1}} \mathrm{~A}_{\mathrm{b}_{1}}, \mathrm{~A}_{\mathrm{a}_{2}} \mathrm{~A}_{\mathrm{b}_{2}}, \ldots, \mathrm{A}_{\mathrm{a}_{\mathrm{N}}} \mathrm{A}_{\mathrm{b}_{\mathrm{N}}}$ of $\mathrm{N}$ successive pairs of the type $\mathrm{A}_{a_{i}} \mathrm{~A}_{b_{i}}$; from each pair there is sue two arrows marked ' $y$ ' and ' $n$ ' and leading to other pairs. We put $A_{0}=S$ and we let in the last pair $a_{N}=b_{N}=0$ interpreting $S S$ as the command 'stop'. In each pair $a_{i}$ and $b_{i}$ are 
non-negative integers or they may be themselves locations. Therefore each command in the string is uniquely determined by an ordered 6-tuple of non-negative integers $\left(p_{i}, q_{i}, r_{i}, s_{i}\right.$, $\left.t_{i}, u_{i}\right)$ in the following way: with $A_{i} A_{j}$ we associate $(i, 0, j, 0, h, k)$, with $A_{A} A_{j}(i, 1, j, 0, h, k)$, with $A_{i} A_{A_{j}}$, $(i, 0, j, 1, h, k)$, and with $A_{A} A_{i},(i, 1, j, 1, h, k)$.

In each case the fifth member $k$ of the 6-tuple shows that the arrow marked ' $y$ ' leads to the $k$-th pair of the string and the sixth member $h$ shows that the arrow marked ' $n$ ' leads to the h-th pair of the string. Now $P$ becomes an ordered set of ordered 6-tuples $\mathrm{S}_{1}, \ldots, \mathrm{S}_{\mathrm{N}}$; let $f:\left(\mathrm{n}_{1}, \mathrm{n}_{2}, \mathrm{n}_{3}, \mathrm{n}_{4}, \mathrm{n}_{5}\right.$, $\left.n_{6}\right) \rightarrow n$ be a function mapping in a 1:1 fashion the set of all ordered 6-tuples of non-negative integers onto the non-negative integers themselves. With the program $P=\left(S_{1}, \ldots, S_{N}\right)$ we now associate the number $N_{P}=\prod_{i=1}^{N} p_{i} f\left(S_{i}\right)$, where $p_{i}$ is the i-th prime, starting with $\mathrm{p}_{1}=2$. Of course, not every number is the number of a program, and a program with the number $\mathrm{N}_{1}$ may be completely equivalent in its effects on all locations to a program with a different number $\mathrm{N}_{2}$. If $P_{1}, P_{2} \in S_{2}^{0}$ we define the composite program $P_{1} P_{2}$ by juxtaposition, with all the terminal arrows of $P_{1}$ leading to the initial command of $\mathrm{P}_{2}$. Letting 1 stand for the empty program which does nothing, the set $\jmath_{2}^{0}$ becomes a countable semigroup with identity.

15. An interesting binary relation on the set of all $Q$-machines is obtained by letting $Q^{a} \leq Q^{b}$ if and only if the command of $Q^{a}$ can be synthesized as a loopless program on $Q^{b}$. Let also $Q^{a}<Q^{b}$ if $Q^{a} \leq Q^{b}$ and $Q^{a} \geq Q^{b}$. For instance, we have $Q_{2}^{0}<Q_{3}^{0}<Q_{5}^{0}$; the reason for this is that the basic operations of the se three machines (adding 1, adding 
two integers, adding and multiplying two integers) are on different levels of a progressive hierarchy of the magnitude of the computed number. We derive now some sufficient conditions for two Q-machines, $Q^{a}=Q_{n}^{a}\left(f_{1}, \ldots, f_{n}\right)$ and $Q_{m}^{\bar{b}}\left(g_{1}, \ldots, g_{m}\right)$, to ensure that $Q^{a}<Q^{b}$. A function $F(x)$ dominates $Q^{a}$ if for every set of non-negative integer arguments $\left(c_{1}, \ldots, c_{n}\right)$

$$
\left|c_{i}+f_{i}\left(c_{1}, \ldots, c_{n}\right)\right|<F\left(\max \left(c_{1}, \ldots, c_{n}\right)\right), \quad i=1, \ldots, n \text {. }
$$

For a positive integer $k$ let $F_{k}(x)$ denote the $k$-th iterate of $F(x)$. We say that $G(x)$ majorizes $F(x)$ (and also that $G(x)$ majorizes $Q^{a}$ ) if for every fixed $k$ there is $x_{0}$, such that $F_{k}(x)<G(x)$ provided that $x_{0}<x$. It is well known that every function $F(x)$, no matter how fast its growth, possesses a majorizing function $\mathrm{G}(\mathrm{x})$. For instance, we can take

$$
G(x)=\sum_{j=1}^{\infty} a_{j} F_{j}(x),
$$

where the sequence $\left\{a_{j}\right\}$ tends to 0 fast enough. Suppose now that a) $Q^{b}$ is an extension of $Q^{a}$, and b) the first transfer function $g_{1}$ of $Q^{b}$ is such that for suitable values $c_{2}, \ldots, c_{m}$ there occurs a transfer of at least $G\left(c_{1}\right)$ counters to some location, $G(x)$ being any function majorizing $Q^{a}$. Condition a) implies $Q^{a} \leq Q^{b}$. Observe next that any loop-free program of $Q^{a}$ with a string of $k$ consecutive commands, and with the initial contents $p_{1}, \ldots, p_{N}$, results in final contents none of which can exceed $F_{k}\left(\max \left(p_{1}, \ldots, p_{N}\right)\right)$. On the other hand, a single command of $Q^{b}$ can, with the same initial contents, produce final contents which exceed $F_{k}\left(\max \left(p_{1}, \ldots, p_{N}\right)\right.$, no matter how large $k$ may be. Therefore condition b) implies $\mathrm{Q}^{\mathrm{b}} \ddagger \mathrm{Q}^{\mathrm{a}}$. It follows that $\mathrm{Q}^{\mathrm{a}}<\mathrm{Q}^{\mathrm{b}}$. Since $\mathrm{Q}^{\mathrm{a}}$ was arbitrary, we can carry on the process to obtain for any initial 
$Q^{1}=Q^{a}$ an infinite chain of the type $Q^{1}<Q^{2}<Q^{3}<\ldots$

By the construction of this chain, for any $n$ the $Q^{n}$ program equivalent to the command of $Q^{n+1}$ contains at least one loop, the $Q^{n}$ program equivalent to the command of $Q^{n+2}$ contains at least one loop-within-a-loop, and generally, the $Q^{n}$ program equivalent to the command of $Q^{n+k}$ contains at least one k-tuply imbedded loop. This shows that no matter which machine is used for computing, there will be numbers computing which calls for programs of arbitrarily high degree of loop imbedding.

16. For the purpose of this section we modify the definition of certain Q-machines so that they can handle negative integers. Consider first $Q_{3}^{0}$; its basic operations are addition of non-negative integers, restricted subtraction of integers ( = formation of the difference $a-b$ when $a \geq b \geq 0$ ) and the conditional transfer which depends on the previous operation. Suppose now that instead of a single row of locations there is a double row

$$
A_{1}^{+}, A_{2}^{+}, \ldots .
$$

and let the contents of $A_{n}$ be the difference (contents of $A_{n}^{+}$) - (contents of $A_{n}^{-}$). This amounts to the usual process of regarding integers as pairs of non-negative integers with the ordinary provision for pair equivalence: $(a, b)=a-b$ and $(a, b)=(c, d)$ if $a+d=b+c$. There is, as before, a single store $S$. The $Q_{3}^{0}$ command $A_{1} A_{2} A_{3}$ is now interpreted as the unbranched program 


$$
\begin{aligned}
& S A_{2}^{-} A_{1}^{+} \\
& S A_{2}^{+} A_{1}^{-} \\
& S A_{2}^{+} A_{3}^{+} \\
& S A_{2}^{-} A_{3}^{-} .
\end{aligned}
$$

Thus modified, $Q_{3}^{0}$ can handle negative integers. However, the ability to perform conditional transfer vanishes, since now subtraction is always possible. Here this ability can be easily restored: replace

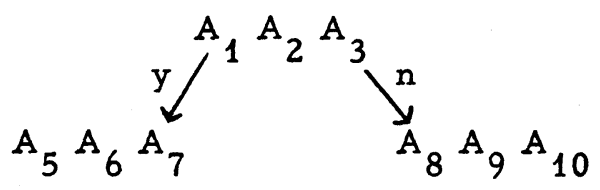

by the program

$$
\begin{aligned}
& \mathrm{SA}_{1}^{+} \mathrm{A}_{4}^{+} \\
& \mathrm{SA}_{2}^{-} \mathrm{A}_{4}^{+} \\
& \mathrm{SA}_{2}^{+} \mathrm{A}_{4}^{-} \\
& \mathrm{S} \mathrm{A}_{1}^{-} \mathrm{A}_{4}^{-} \\
& \mathrm{A}_{1}^{+} \mathrm{A}_{1}^{-} \mathrm{A}_{3}^{-} \\
& \mathrm{A}_{4}^{+} \mathrm{A}_{4}^{+} \mathrm{A}_{1}^{+} \\
& \mathrm{A}_{5} \mathrm{~A}_{6} \mathrm{~A}_{7}^{+}
\end{aligned}
$$


Let $\bar{Q}_{3}^{0}$ denote the above modification of $Q_{3}^{0}$ to handle negative integers. It is clear that with four rows of locations we can further modify $Q_{3}^{0}$ so that it can handle Gaussian integers.

In this section there will be proved a theorem, formulated in terms of the Q-machines, but having some independent interest. It is given here as an example of some uses of our theory. The question which led to it, was: what is the simplest way of computing?

In the first part of this paper we considered the sequence $\left\{p_{n} / q_{n}\right\}$ of fractions, $n=0,1, \ldots$, with $p_{0}>0, q_{0}>0$ and with the recursion formula $p_{n+1}=p_{n}+2 q_{n}, q_{n+1}=p_{n}+q_{n}$. One has then $\lim _{n \rightarrow \infty}\left(p_{n} / q_{n}\right)=+2^{1 / 2}$, and an unbranched $Q_{3}^{0}$ program was given to compute each successive convergent fraction from the previous one by means of four additions of positive integers and no other operations. Since the addition of integers is one of the simplest possible operations, this raises the question: which (real) numbers can be computed by means of iterative schemes with a bounded number of additions of integers per iteration stage and no other operations?

The above formulation is not yet precise enough, and to emend it we define a real number $x$ to be $\bar{Q}_{3}^{0}$ computable if there exists an unbranched $\bar{Q}_{3}^{0}$ program:

$$
\begin{aligned}
& \text { initial contents: } a_{10}, \ldots, a_{k 0}, 0, \ldots \\
& \text { program: } \\
& \left(\begin{array}{ccc}
A_{i_{1}} & A_{j_{1}} & A_{k_{1}} \\
{ }_{{ }_{i p}} & A_{j_{p}} & A_{k}
\end{array}\right.
\end{aligned}
$$

which is non-terminating and which results in accumulating successively in certain two locations, say in $A_{1}$ and $A_{2}$, 
of two sequences of integers, $\left\{p_{n}\right\}$ and $\left\{q_{n}\right\}, p_{n}$ and $q_{n}$ being the contents after the $n$-th cycle, such that $\lim \left(p_{n} / q_{n}\right)=x$. The sequences $\left\{p_{n}\right\}$ and $\left\{q_{n}\right\}$ will be called admissible for x. Our question is now re-formulated: which numbers are $\overline{\mathrm{Q}}_{3}^{0}$ computable? A partial answer is given in the following Theorem. Any real $\overline{\mathrm{Q}}_{3}^{0}$ computable number is algebraic. If $\mathbf{x}$ is a PV-number then any number of the form $\left(t_{1} x+t_{2}\right) /\left(t_{3} x+t_{4}\right)$, with the $t_{i}{ }^{\prime}$ s all integers, is $\bar{Q}_{3}^{0}$ computable.

Recall that the PV-numbers (Pisot-Vijayaraghavan numbers) are real positive algebraic integers greater than 1 , all of whose other conjugates are less than 1 in absolute value. For their properties see [4].

The idea of the proof is to show first that every computation method for a $\bar{Q}_{3}^{0}$ computable number $x$ is essentially the same as the above special case of the sequences $\left\{p_{n}\right\}$ and $\left\{q_{n}\right\}$ which are admissible for $+2^{1 / 2}$ : there is a linear system of recurrence relations for a finite number of sequences $\left\{p_{n}\right\},\left\{q_{n}\right\}, \ldots,\left\{z_{n}\right\}$, and $x$ is the limit of a ratio, say $\left(p_{n} / q_{n}\right)$. We observe first that since there are no subscripted Iocations $\mathrm{A}_{\mathrm{A}_{i}}$, only a finite number of locations is ever occupied throughout the program. It may be assumed therefore that the locations $A_{k+1}, A_{k+2}, \ldots$ stay empty throughout.

Let $a_{\text {in }}, i=1, \ldots, k$, be the contents of the $i-$ th location after the $n$-th cycle. Since each successive command of the program results in location contents which are linear combinations of the contents before the command, we have

(4) $\quad a_{i n+1}=\sum_{j=1} c_{i j} a_{j n}, i=1, \ldots, k ; a_{10}, \ldots, a_{k 0}$ given integers. 
The integers $c_{i j}$ are constants independent of $n$ since there are no subscripted locations. Solution of the system (4) is obtained by assuming that

$$
a_{\text {in }}=\sum_{s=1}^{k} b_{\text {is }} \lambda_{s}^{n}, \quad i=1, \ldots, k ; n=0,1, \ldots
$$

on substituting (5) into (4) and equating the coefficients of each $\lambda_{s}^{n}$ one finds that $\lambda_{1}, \ldots, \lambda_{k}$ are the eigenvalues of the matrix $\left(c_{i j}\right)$. Therefore the procedure is justified provided that the eigenvalues $\lambda_{1}, \ldots, \lambda_{k}$ are all distinct. Otherwise one has instead of (5)

(6) $\quad a_{\text {in }}=\sum_{s=1}^{K} B_{i s}(n) \lambda_{s}^{n}, \quad i=1, \ldots, k ; n=0,1, \ldots$;

here $\lambda_{s}$ is an eigenvalue of multiplicity $\mathrm{n}_{\mathrm{K}}, \mathrm{B}_{\text {is }}(\mathrm{n})$ is a polynomial in $n$ of degree $n_{s}-1$ and $\underset{s=1}{\sum_{s}} n_{s}=k$. The coefficients of the polynomials $B_{\text {is }}$ are obtained on substituting (6) into (4) and equating the coefficients of $n^{p} \lambda_{s}^{n}$ for each pair p, s. In either case, whether the eigenvalues are simple or not, the coefficients $b_{\text {is }}$ or the coefficients of the polynomials $B_{\text {is }}$ are obtainable by algebraic processes from the integers $c_{i j}$ and $a_{i 0}$ and the eigenvalues $\lambda_{i}$, which are algebraic numbers. Therefore these coefficients are themselves algebraic. If $x$ is a $\bar{Q}_{3}^{0}$ computable number then by definition $x=\lim _{n \rightarrow \infty}\left(a_{i n} / a_{j n}\right)$, so $x$ is either some ratio $b_{i s} / b_{j s}$ or the ratio of the leading coefficients in certain two polynomials $B_{i s}$ and $B_{j s}$. In either case $\mathbf{x}$ is algebraic.

Suppose next that $x$ is $\bar{Q}_{3}^{0}$ computable with the admissible sequences $\left\{F_{n}\right\}$ and $\left\{q_{n}\right\}$. Therefore, if the $t_{i}$ 's 
are integers, the number $\left(t_{1} x+t_{2}\right) /\left(t_{3} x+t_{4}\right)$ is also $\bar{Q}_{3}^{0}$ computable with the admissible sequences $\left\{t_{1} p_{n}+t_{2} q_{n}\right\}$ and $\left\{t_{3} p_{n}+t_{4} q_{n}\right\}$. It remains to show that a $P V$-number is $\bar{Q}_{3}^{0}$ computable. The integers $c_{i j}$ in (4) are arbitrary - let them be chosen so that the given PV-number $x$ is one of the eigenvalues $\lambda_{s}$ of the matrix $\left(c_{i j}\right)$. Then all the other eigenvalues are less than $x$ in absolute value, so that

$$
x=\lim _{n \rightarrow \infty}\left(a_{1 n+1} / a_{1 n}\right)=\lim _{n \rightarrow \infty}\left[\left(\sum_{s=1}^{k} b_{i s} \lambda_{s}^{n+1}\right) /\left(\sum_{s=1}^{k} b_{\text {is }} \lambda \lambda_{s}^{n}\right)\right]
$$

and hence the sequences $\left\{a_{1 n+1}\right\}$ and $\left\{a_{1 n}\right\}$ are admissible for $x$. Thus $x$ is $\bar{Q}_{3}^{0}$ computable.

One can similarly consider the $\bar{Q}_{5}^{0}$ computable numbers. It is first necessary to modify $Q_{5}^{0}$ to operate with negative numbers; this can be arranged without difficulty, as for $Q_{3}^{0}$. Since only unbranched programs will be considered, there is no need to restore the conditional transfer (although this could be done easily). We define a real number to be $\bar{Q}_{5}^{0}$ computable in the same way as before, replacing $\bar{Q}_{3}^{0}$ by $\bar{Q}_{5}^{0}$. Since $\bar{Q}_{5}^{0}$ can add and multiply, instead of the linear combinations of (4) we have now polynomial combinations

$$
a_{i n+1}=P_{i}\left(a_{1 n}, \ldots, a_{k n}\right), i=1, \ldots, k ; n=0,1, \ldots \text {, }
$$

and the coefficients of the polynomials $P_{i}$ are constants independent of $n$ since there are no subscripted locations $\mathrm{A}_{A_{i}}$ in the program. It follows that our $\bar{Q}_{5}^{0}$ computable numbers coincide with the rationally recursive numbers introduced and investigated in [3]. Among other open questions posed in [3] there are: 1) is every rationally recursive number computable?, 
and 2) can one exhibit a computable number which is not rationally recursive (the existence of such is easy to prove)?

From the equation 'rationally recursive' $={ }^{\prime} \bar{Q}_{5}^{0}$ computable' it follows at once that the answer to 1) is yes. Starting with the remarks at the end of the previous section, it is not hard to exhibit a computable number which is not $\bar{Q}_{5}^{0}$ computable. For instance, let

${ }^{2}(1)(x)=2^{x}, 2_{(n+1)}(x)=2^{2}(n)^{(x)},(k !)_{(1)}=k !,(k !)_{(n+1)}=\left[(k !)_{(n)}\right] !$

and define

$$
F(x)=\sum_{n=1}^{\infty} 2(n)(x) /(n !)_{(n)} ;
$$

then for every positive integer $m$ the number $F(m)$ is computable but not $\overline{\mathrm{Q}}_{5}^{0}$ computable.

The author wishes to thank the referee for improvements, corrections and suggestions.

\section{REFERENCES}

1. Z.A. Melzak, An Informal Arithmetical Introduction to Computability and Computation, Can. Math. Bull., vol. 4, no. 3, Sept. 1961.

2. A. M. Turing, On Computable Numbers with an Application to the Entscheidungsproblem, J. Lond. Math. Soc., Spring 1936.

3. H.S. Shapiro, Rational Recurrence Formulae, Comm. Pure Appl. Math. , vol.12, no. 3, Aug. 1959.

4. J.W.S. Cassels, An Introduction to Diophantine Approximation, Cambridge Univ. Press, 1957.

The University of British Columbia 